\title{
In Situ TEM Etching of Gold Nanocrystals: Elucidating the Shape Transformation Mechanisms and Chemistry of the Graphene Liquid Cell
}

\author{
Matthew R. Hauwiller ${ }^{1}$, Justin Ondry ${ }^{1}$, and A. Paul Alivisatos ${ }^{1,2,3,4}$ ** \\ 1. Department of Chemistry, University of California-Berkeley, Berkeley, California, United States. \\ 2. Department of Materials Science and Engineering, University of California-Berkeley, Berkeley, \\ California, United States. \\ 3. Materials Sciences Division, Lawrence Berkeley National Laboratory, Berkeley, California, United \\ States \\ 4.Kavli Energy NanoScience Institute, University of California Berkeley and Lawrence Berkeley \\ National Laboratory, Berkeley, California, United States \\ * Corresponding author: paul.alivisatos@berkeley.edu
}

Liquid cell electron microscopy has the necessary spatial resolution to view nanoscale dynamics in their native liquid environment. The ability to image colloidal nanocrystal processes in solution has revealed a significant amount of information about the formation and interaction of nanocrystals. For these insights to be useful for materials scientists, the processes viewed in the microscope must be reproducible with well-understood chemistry. Unfortunately, radiolysis of the liquid solution by the electron beam and inability to measure the concentrations of the generated reactive species renders it difficult to understand the chemistry of the processes being studied. Through the development of a model nanocrystal etching system where we can quantify the size and shape of the etching nanocrystals, we have been able to better understand the chemistry of the liquid cell and learn new insights about nanocrystal etching transformations.

Graphene liquid cell electron microscopy encapsulates a liquid solution containing species of interest between sheets of graphene and images the sample with the high spatial resolution of the electron microscope. [1] This method for encapsulating the liquid has a low barrier to entry for new research groups with few fabrication steps and low start-up costs. [2] Gold nanocrystals were etched using a combination of the electron beam-generated radiolysis species and preloaded $\mathrm{FeCl}_{3}$. The etching occurred in a highdriving force regime generating non-equilibrium intermediate shapes. [3] Both cubes with $\{100\}$ initial facets and rhombadodecahedra with $\{110\}$ initial facets transform to $\{$ hk0 $\}$ tetrahexahedra shapes, but modulating the $\mathrm{FeCl}_{3}$ concentration only controls the intermediate steady facets when etching from the cubes. Using Monte Carlo simulations to corroborate the liquid cell observations, the concentration of $\mathrm{FeCl}_{3}$ was found to be the chemical potential in the system, determining which atoms can be removed. [4] Understanding the effect of chemical potential on etching process in the regime of high driving force is useful for synthesizing energetically unfavourable shapes.

Following the volumes of the nanocrystals during the etching process at different electron beam dose rates provides clues to the effect of the electron beam on the chemistry of the water solution. Initially, hydrogen bubbles are generated when looking for nanocrystals at low dose rates, providing a sacrificial reductant that sets a time zero when the electron beam dose rate is increased to the etching dose rate. Once the nanocrystals begin etching, they etch at a constant rate that is linearly dependent on the electron beam dose rate. We are able to independently tune the etching rate and chemical potential of the liquid cell by modulating the electron beam dose rate and initial $\mathrm{FeCl}_{3}$ concentration respectively. The etching transformations in this high driving force regime were correlated with ex situ etching experiments to 
confirm that the etching mechanisms seen in the liquid cell are still relevant for etching processes not induced by the electron beam.

With this high level of control in the etching of gold nanocrystals in the graphene liquid cell, we are able to expand to other important parameters such as the effect of ligands on the etching process. By exchanging the ligands on the nanocrystals prior to encapsulation in a liquid cell, the etching rate and intermediate shape can be modulated. Unique geometries of nanocrystals can be formed through selective binding of ligands to specific facets and positions on the nanocrystal. This investigation reveals that ligands are not destroyed under electron beam irradiation, making graphene liquid cell electron microscopy a promising avenue for studying nanocrystal ligands.

For the liquid cell electron microscopy technique to become a staple in nanomaterials research, the ability to perform reproducible, targeted reactions is needed. Through this work's understanding of the chemistry of the liquid environment and use of that control to elucidate nanocrystal etching mechanisms, we are one step closer to liquid cell electron microscopy becoming a cornerstone materials characterization technique. [5]

\section{References:}

[1] Yuk, J. M. et al, Science 336 (2012), p. 61-64

[2] Hauwiller, M. R et al J. Vis. Exp. 135 (2018), p. 1-9.

[3] Ye, X et al. Science 354 (2016), p. 874-877.

[4] Hauwiller, M. R. et al Nano Letters 18 (2018), p. 5731-5737.

[5] The authors acknowledge funding from the U.S. Department of Energy, Office of Science, Office of Basic Energy Sciences, Materials Sciences and Engineering Division, under Contract No. DEAC02-05CH11231 within the Physical Chemistry of Inorganic Nanostructures Program (KC3103). Professor Phillip Geissler, Professor Xingchen Ye, Professor Matt Jones, and Layne Frechette are thanked for their contributions to this work.
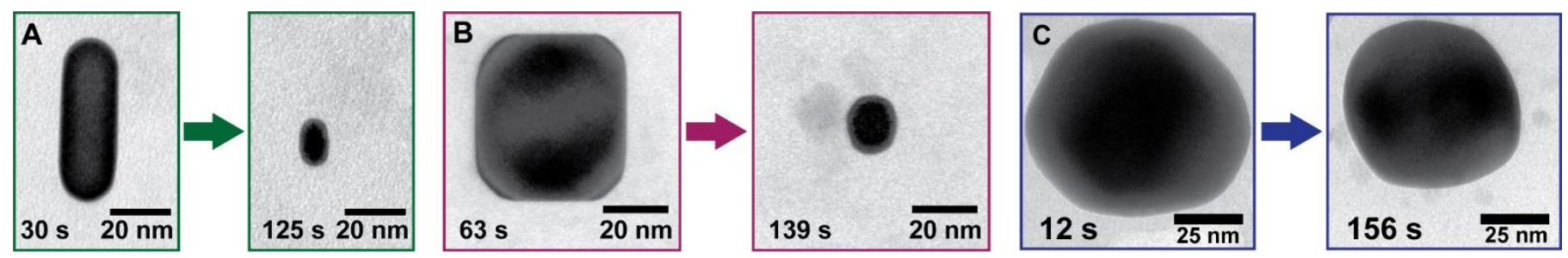

Figure 1. Etching of gold nanocrystals using Graphene Liquid Cell electron microscoopy (A) Etching gold nanorods (B) Etching gold nanocubes with $\{100\}$ initial facets (C) Etching gold rhombadodecahedra with $\{110\}$ initial facets 